\title{
Herramientas didácticas para el desarrollo técnico musical del guitarrista clásico a través de ejes - problemas*
}

\author{
DIDACTIC TOOLS FOR THE MUSICAL AND TECHNICAL DEVELOPMENT OF THE \\ CLASSICAL GUITAR PLAYER THROUGH PROBLEM AXES
}

FERRAMENTAS DIDÁTICAS PARA O DESENVOLVIMENTO TÉCNICO MUSICAL DO VIOLONISTA CLÁSSICO ATRAVÉS DE PROBLEMAS-EIXO

\section{Carlos Posada Ramos** \\ César Augusto Quevedo Barrero*** Andrés Samper Arbeláez****}

\footnotetext{
Cuadernos de Música, Artes Visuales y Artes Escénicas

/ Volumen 11 - Número 1 / enero - junio de 2016

/ ISSN 1794-6670/ Bogotá, D.C., Colombia / pp. 39-53

Fecha de recepción: 1 de julio de 2015 | Fecha de

aceptación: 7 de noviembre de 2015 | Disponible en

línea: 30 de mayo de 2016. Encuentre este artículo en

http://cuadernosmusicayartes.javeriana.edu.co/

doi:10.11144/Javeriana.mavae11-1.hddt
}

* Artículo de investigación. Parte de la investigación Herramientas didácticas para el desarrollo técnico musical del guitarrista clásico a través de ejes - problemas, del Departamento de Música de la Pontificia Universidad Javeriana.

* Maestro de la Universidad de Viena. Profesor Asociado del Departamento de Música de la Pontificia Universidad Javeriana de Bogotá

*** Maestro del Koninklijk Conservatorium - Conservatorio Real de La Haya. Profesor Instructor del Departamento de Música de la Pontificia Universidad Javeriana de Bogotá.

**** Doctorando en Educación Musical en el Instituto de Educación del University College of London. Profesor Asistente del Departamento de Música de la Pontificia Universidad Javeriana de Bogotá. 


\section{Resumen}

Este artículo presenta los resultados de un estudio desarrollado por el área de guitarra clásica del Departamento de Música de la Pontificia Universidad Javeriana (PUJ) en Bogotá, Colombia. El estudio indagó sobre las dificultades técnico-musicales más recurrentes entre estudiantes de guitarra de los programas infantil y juvenil, pregrado y posgrado de la PUJ. Con base en las dificultades detectadas se realizó una recopilación de material musical para apoyar el desarrollo de habilidades en la formación del guitarrista clásico. El material pedagógico resultante hace énfasis en el desarrollo musical, plantea una estructura no lineal de aprendizaje, incorpora el uso de materiales latinoamericanos e incluye ejercicios de improvisación, lectura a primera vista y grafías no convencionales. La metodología empleada fue de carácter cualitativo y se fundamentó en un ejercicio triple de reflexión didáctica, sistematización de materiales existentes y creación de piezas originales.

Palabras clave: enseñanza de la guitarra; didáctica instrumental; material pedagógico para guitarra; método para guitarra; guitarra clásica

\section{Abstract}

This paper presents the results of a study undertaken by the classical guitar area of the Music Department at Pontificia Universidad Javeriana (PUJ) in Bogotá, Colombia. The study enquired into emergent difficulties among guitar students of the youth, undergraduate and graduate programs at PUJ. On the basis of the difficulties that were detected a compilation of musical material was developed that can be used as support for the training of classical guitarists. The final proposal of pedagogic material emphasises musicality, is built upon a non-linear structure for learning and incorporates Latin American pieces as well as exercises that deal with improvisation, first-sight reading and non-conventional notation. A qualitative type of methodology was used based upon a three-fold exercise of didactic reflection, systematization of existing musical material and creation of original pieces.

Keywords: teaching of guitar; music didactics; pedagogic material for guitar; method for guitar; classical guitar

\section{Resumo}

O presente artigo apresenta os resultados de um estudo desenvolvido pela área de violão clássico do Departamento de Música da Pontificia Universidad Javeriana (PUJ) em Bogotá, Colômbia. O artigo inquiriu sobre as dificuldades técnico - musicais mais recorrentes entre os alunos de violão do Programa Infantil e Juvenil, da graduação e pós graduação da PUJ. Baseados nas dificuldades detectadas, foi realizada uma recopilação de material musical para ajudar o desenvolvimento de habilidades na formação do violonista clássico. O material pedagógico resultante faz uma ênfase no desenvolvimento musical, planteia uma estrutura não lineal de aprendizagem, incorpora a utilização de materiais latino americanos e insere exercícios de improvisação, leitura à primeira vista e grafias não convencionais. A metodologia empregada foi de caráter qualitativo y fundamentou-se num exercício triplo: de reflexão didática, sistematização dos matérias existentes e criação de peças originais.

Palavras chave: pedagogia do violão; didática instrumental; material pedagógico para violão; método para violão; violão clássico 


\section{INTRODUCCIÓN}

El uso de herramientas didácticas para facilitar el desarrollo del estudio de la guitarra clásica es un tema abordado por muchos autores a través de la historia del instrumento. Desde los primeros métodos de estudio e instrucción desarrollados por los vihuelistas del siglo XVI hasta hoy, podemos encontrar una gran cantidad de material disponible en este sentido. En línea con esta tradición, este proyecto proporciona una recopilación de herramientas y materiales musicales que apoyen el desarrollo de habilidades y saberes específicos a lo largo del proceso de formación del guitarrista clásico. El presente artículo da cuenta de los resultados finales del proyecto.

La metodología empleada fue de carácter cualitativo y se fundamentó en un ejercicio triple de reflexión didáctica, sistematización de materiales existentes y creación de productos musicales nuevos con propósitos pedagógicos. El estudio fue desarrollado por tres profesores del área de guitarra clásica del Departamento de Música de la Facultad de Artes en la Pontificia Universidad Javeriana (PUJ) en Bogotá, con una experiencia pedagógica de más de veinte años. La motivación del estudio surgió de un interés por potenciar el desarrollo técnico y musical de los estudiantes de guitarra de la PUJ. En este sentido, el proyecto implicó una etapa inicial en la que se hizo un rastreo de las principales falencias y vacíos que el equipo de profesores ha detectado a lo largo de su experiencia enseñando la guitarra y a través de la investigación misma, en estudiantes de los programas infantil y juvenil, pregrado y posgrado de la PUJ. También fue una motivación la idea de sistematizar de una manera juiciosa las herramientas (ejercicios, estudios, piezas, entre otros) que el área de guitarra ha venido recopilando de manera informal a lo largo de los años. El resultado final del proyecto, al cual se hará alusión detallada más adelante, es un compendio de material pedagógico (ejercicios y piezas) elaborado a partir de las falencias detectadas en el diagnóstico inicial.

Las herramientas que ofrece el proyecto, las opciones y direcciones que propone, los ejemplos que cita, la bibliografía que recomienda y el material original que proporciona, permiten que el proyecto haga aportes al campo de la enseñanza de la guitarra en los siguientes sentidos: en primer lugar, en cuanto a la manera de afrontar los problemas técnicos desde una perspectiva musical, es decir, integrando la mecánica simple del movimiento físico con la aplicación de herramientas musicales como volumen, timbre y velocidad. De esta forma, el proyecto "Herramientas didácticas para guitarristas" es una propuesta que aporta soluciones para una formación integral del músico guitarrista, toda vez que busca no disociar el desarrollo técnico del musical.

En segundo lugar, una de las características importantes del proyecto es que es abordable para cualquier guitarrista en cualquier punto de su formación, desde básico hasta avanzado Es decir, será útil para profesores, alumnos y autodidactas. Se trata de una propuesta que no está estructurada, como se verá más adelante en este artículo, a través de etapas lineales (como sucede en la mayor parte de los métodos tradicionales) sino a partir de problemas específicos para los cuales se propone un corpus de material pedagógico con una diversidad de niveles y de estilos. Se podría decir, en este sentido, que el producto final funciona más como una "red" que como una secuencia lineal de materiales. Este tipo de producto "abierto", que queda documentado en un site de internet, permite además que la ampliación de sus contenidos siga activa a través del tiempo, mediante la retroalimentación constante por parte del equipo de autores del proyecto como también de colegas de otras instituciones y espacios de formación. 
Un tercer aspecto clave es el énfasis que se ha hecho en recolectar material producido para la guitarra clásica en América Latina, en especial por parte de guitarristas y compositores del Departamento de Música de la PUJ. Finalmente, el proyecto indaga sobre ciertos aspectos que aún son poco comunes en este tipo de propuestas pedagógicas, según nos lo mostró la revisión de literatura, como son el tema de la lectura a primera vista, la improvisación y el trabajo con grafías no convencionales.

\section{ALGUNOS ANTECEDENTES}

Una revisión de los textos y métodos escritos para guitarra, de carácter didáctico, nos lleva inicialmente a los compositores de la primera mitad del siglo XIX, momento de oro para la guitarra clásica. Encontramos en ese periodo autores como Ferdinando Carulli (1770-1841), Mateo Carcassi (1792-1853), Mauro Giuliani (1781-1829) y Fernando Sor (1778-1839). Se puede afirmar que estos autores fueron pioneros en el campo de la producción pedagógica para el instrumento, pues existen pocos antecesores en esa área: principalmente casos aislados, la mayor parte provenientes de la literatura para laúd, vihuela y guitarra barroca. Más tarde, la guitarra romántica evolucionó y los autores mencionados dirigieron sus esfuerzos a plasmar en los métodos su experiencia, su propia manera de entender el proceso técnico-musical y las capacidades expresivas del instrumento, enmarcados dentro del estilo musical y las tendencias propias del Romanticismo europeo del siglo XIX. La producción pedagógica de estos autores se vio recogida algunas décadas más tarde por Francisco Tárrega (1852-1909). Sus propuestas en cuanto a sonido, repertorio y técnica fueron decisivas. Este es un punto de articulación entre los siglos anteriores y la entrada al siglo XX. Personalidades posteriores como Miguel Llobet (1878-1938) y Emilio Pujol (1886-1980), ambos alumnos de Tárrega, también emprendieron la labor de ampliar el repertorio con transcripciones de otros autores no guitarristas.

Los métodos estaban generalmente organizados en torno al desarrollo de habilidades técnicas específicas como escalas y arpegios, entre otras. Era común encontrar en el material un ordenamiento de lo fácil a lo complejo y algunos de ellos organizaron sus métodos en torno a una estructura "por tonalidades" es decir, dedicando una sección a cada una de las tonalidades que se pueden interpretar en el instrumento. Sin excepción, incluyeron en sus métodos composiciones originales con propósitos pedagógicos, tanto ejercicios como estudios. También se incluían obras cortas y adaptaciones de composiciones de otros autores.

Vale la pena mencionar que Pujol realiza una sistematización muy importante del modo de enseñar con la Escuela razonada de la guitarra (1954); en este trabajo, el autor recoge aspectos relevantes de las técnicas y metodologías desarrolladas porTárrega, y plantea un razonamiento novedoso para aquel momento acerca del quehacer didáctico con el instrumento.

Varios de estos trabajos, sin embargo, se centran en plantear alternativas únicas para solucionar los problemas técnicos. Un ejemplo muy concreto de esto lo encontramos cuando Pujol, en su libro II de la Escuela razonada de la guitarra, brinda indicaciones para pulsar las cuerdas planteando un modo único de atacar las cuerdas, "vetando" la acción de repetir un mismo dedo en la digitación de mano derecha $(i, m$,a). Cuando en realidad, repetir dedos

puede ser de hecho una herramienta muy útil desde el punto de vista expresivo y tímbrico en determinados contextos musicales. En un sentido semejante, se puede observar en el libro Método Moderno (1921) de Pascual Roch (1860-1921), alumno también de Tárrega, cómo se 
indica solamente una manera de producir el sonido con el pulgar, lo cual resulta limitado desde el punto de vista tímbrico, en especial para la interpretación de repertorio contemporáneo.

Hasta la época de Pujol y Llobet se puede observar una tendencia marcada a teorizar acerca del desarrollo técnico, sin explicaciones sólidas que ayuden a unir el plano meramente técnico con los fines musicales y sin implicaciones de carácter fisiológico que tengan en cuenta las diferencias propias de cada estudiante.

Avanzando en esta dirección, encontramos el trabajo de Abel Carlevaro (1916-2001), quien representa un ícono de la técnica en el s. XX y cuya obra más conocida es el libro Escuela de la Guitarra - Exposición de la teoría instrumental (2006) que complementa la Serie Didáctica para Guitarra (4 volúmenes). El trabajo de Carlevaro es ampliamente apreciado en el mundo guitarrístico, por el agudo nivel de sistematización de su propuesta para desarrollar mecanismos conducentes a dominar a un nivel alto los problemas técnicos. Aun así, la propuesta de Carlevaro de agrupar masivamente ejercicios dirigidos a trabajar algunas dificultades técnicas es susceptible de ser revisada. Existen numerosas combinaciones en las digitaciones, presentes en obras del siglo XX y obras de la literatura guitarrística posteriores a Carlevaro, que fueron excluidas. En su primer tomo, Escalas diatónicas, no parece existir una relación entre sus modelos de escalas y los otros tomos de su trabajo; aún más, los modelos de escalas parecen planteados como si fueran la única posibilidad de realizar las mismas, desconociendo abiertamente la gran cantidad de posibilidades que tiene el instrumento. Tampoco se puede apreciar una relación muy clara entre estos modelos de escalas diatónicas y el repertorio vigente hasta la época de Carlevaro. Finalmente, más allá del invaluable valor reflexivo del ejercicio sistemático realizado por Carlevaro y de su potencial pedagógico en cuanto al desarrollo de la mecánica instrumental, se puede evidenciar una reiteración de la separación técnica-música; disociación que este proyecto busca contribuir a superar.

Otro referente importante consultado por este proyecto son los trabajos de Mario Gangi (1923-2010) y Aaron Shearer (1919-2008). Los escritos de Gangi son más propios de un compositor y sus 22 estudios (1971) así lo evidencian; en ese sentido, los dos primeros libros del autor (Metodo per Chitarra, prima parte y seconda parte, 1971) son una preparación directa para abordar estos estudios. Gangi está influenciado de modo notable por la herencia italiana de Carulli, Giuliani y Legnani, entre otros.

Por su lado, Shearer ha dejado un extenso y detallado método para guitarra (Learning the classic guitar, part one and part two, 1990), de corte muy tradicional, que presenta explicaciones claras que sin duda están marcadas por la experiencia personal de una lesión corporal que afectó su interpretación de la guitarra. El trabajo de Shearer es relevante para el presente trabajo, ya que considera lo orgánico y natural en la mecánica, cuando es utilizado con fines musicales.

Por último, el ensayo del guitarrista Eduardo Fernández "Técnica, mecanismo y aprendizaje" (año 2000) sirve al presente proyecto como un punto de partida dado que enuncia de modo claro la relación que debe haber entre la idea musical, el análisis y la realización de tales resultados en la interpretación del instrumento.

Finalmente, es importante señalar que el estudiante que está enfrentado a materiales extensos de técnica y repertorio, puede sentir una desorientación estética. Un desligamiento entre su posición actual como individuo cultural comprometido con la música, y los espacios en donde se transmiten, socializan y visibilizan los repertorios de la guitarra clásica. Los métodos más aceptados en el contexto mundial para el aprendizaje y práctica de la guitarra clásica 
aparecen como propuestas inflexibles y rígidas en varios puntos. Son métodos lineales y homogenizantes en muchos aspectos, pues dejan a un lado las diferencias sutiles que existen entre un estudiante y otro, y por ende, entre un intérprete y otro.

Aun cuando se debe resaltar la gran labor de organización que ofrecen métodos sobresalientes como los mencionados arriba, el presente proyecto ofrece una perspectiva alternativa que procura trascender estas miradas lineales del aprendizaje, al tiempo que abre las puertas a temas como la improvisación, la composición, la lectura a primera vista y a una multiplicidad de efectos sonoros en la guitarra. Esto, unido a una presencia importante de repertorio latinoamericano, permite construir relaciones más significativas y directas entre los componentes de la formación del guitarrista, y las características y exigencias del medio musical contemporáneo en el que se van a desarrollar y presentar sus propuestas estéticas.

\section{HACIA UNA PROPUESTA ALTERNATIVA DEL “MÉTODO” INSTRUMENTAL}

La enseñanza instrumental al interior de la academia ha tenido desde siempre al "método" como soporte didáctico por excelencia. Se trata de una construcción pedagógica típica de la modernidad que estructura el conocimiento de una determinada disciplina dentro de una línea de contenidos secuenciales, organizados por etapas de creciente dificultad.

Durante mucho tiempo se mantuvo una visión del método como proceso lineal, rígido y secuenciado que impregnó las prácticas de enseñanza, inscribiéndose en una concepción de ciencia que "niega el conjunto de relaciones que inciden en los procesos de conocimiento" (Edelstein en Camilloni, 1998, p. 79). Esta forma de ver el método, fuertemente arraigada en disposiciones universales, tiene como consecuencia una priorización de la prescripción.

Este tipo de enfoque se evidencia en la producción pedagógica para guitarra a la que se hace alusión en la sección anterior de este artículo. Sin embargo, es interesante observar que la idea en torno a lo metodológico como un proceso secuenciado y prescriptivo sigue hoy en día presente en las prácticas de enseñanza instrumental. Es común, de hecho, utilizar el "método" de algún compositor(es) o instrumentista(s) reconocido(s) como herramienta de trabajo en el proceso de formación instrumental. Usualmente el método recoge objetivaciones de situaciones y aspectos técnico-musicales extraídos de su contexto para abordarlos de manera aislada y facilitar así la comprensión de los mismos, con la ayuda de ejercicios, estudios y/o fragmentos de piezas del repertorio. La utilización del método no es, por supuesto, en sí misma problemática; lo que a nuestro juicio plantea dificultades es cuando la enseñanza se queda "atrapada" en un solo método, y por lo tanto, en un solo corpus de repertorios y estilos. Con todas las limitantes que esto implica respecto a la versatilidad en la formación, por una parte, y por otra, respecto a la posibilidad de sintonizar mejor con el universo estético y los intereses de los estudiantes.

Frente a esta visión monolítica del método, Edelstein desarrolla, a partir de la revisión de los planteamientos de algunos autores contemporáneos, una propuesta que entiende lo metodológico como acción creativa y de construcción del docente, quien a partir de los cuerpos de conocimientos propios de su disciplina, los contextos, y la dimensión axiológica de su propia práctica, realiza sus elaboraciones metodológicas propias. Esta construcción involucra al docente en un: 
(...) acto 'singularmente creativo' en el cual se asume como intelectual que elabora y sostiene

una propuesta en la que proyecta sus adscripciones teóricas y axiológicas, su biografía escolar

y su trayectoria profesional. (Edelstein, 2014, p. 49)

En este sentido, la propuesta del presente estudio rescata la idea de "método" desde una perspectiva más flexible, que atiende a necesidades de cada intérprete en particular y que ofrece como producto una serie de ejercicios y posibilidades de repertorio entre los cuales el docente puede "navegar" de manera libre, de acuerdo a las necesidades puntuales de su práctica pedagógica y a los perfiles de sus estudiantes.

En este marco de ideas, en este estudio se desarrolló una estructura no lineal, que atiende a una visión holística del aprendizaje de la música, que va del todo a las partes y de las partes al todo. Se plantea en este sentido, que las estructuraciones fuertes en segmentos lineales de los métodos tradicionales presentan limitaciones en cuanto suelen dar por hecho que el desarrollo técnico-musical es acumulativo y homogéneo para todos los estudiantes; cuando en realidad cada alumno es un universo de particularidades inserto en procesos no lineales de desarrollo que tienen que moverse de manera cíclica hacia adelante y hacia atrás para fortalecer el desarrollo de las diversas competencias.

En otras palabras, el hecho de que un estudiante concluya el primer libro de un método, por ejemplo, no implica que haya necesariamente desarrollado todas las habilidades técnicas y musicales que este "nivel" buscaba desarrollar, de manera plena y absoluta. Siempre habrá una circularidad en el proceso que implicará seguir fortaleciendo o afianzando dichas habilidades. Volver sobre las lecciones ya "aprendidas" trae consigo siempre un punto de vista diferente y renovado, que brinda nuevas posibilidades de apreciación del mismo material y por consiguiente, una nueva visión del desarrollo personal. En esta línea, el producto de este estudio ofrece un compendio de ejercicios y repertorios organizados a partir de núcleos problemáticos que podrán ser abordados en cualquier orden y en cualquier momento del desarrollo instrumental como soporte a los procesos de aprendizaje de la guitarra.

De otra parte, se plantea la importancia de atender en los procesos de aprendizaje musical a la inclusión de repertorios que representen una gama más amplia de lenguajes y estilos. Los currículos tradicionales de enseñanza musical han estado fuertemente dominados por la música centro europea; sin embargo, es innegable que el contexto contemporáneo exige una sensibilización de los instrumentistas frente a diversos géneros y estilos, que les ofrezcan elementos para ser más versátiles en el contexto, pero que además hagan más significativas las prácticas de enseñanza acercándolas a las estéticas musicales de los jóvenes, como el blues, el jazz, el rock y otras expresiones de música popular urbana.

En este mismo sentido, se ha incluido dentro de los repertorios abordados elementos y piezas del repertorio popular colombiano, entendiendo que la PUJ está inserta dentro de un contexto cultural específico del cual es a la vez parte y agente dinamizador. Así, la inclusión de músicas colombianas tradicionales en los repertorios, construye perfiles de músicos más conectados con el contexto, y a la vez preserva y dinamiza la tradición cultural.

Por último, se plantea la importancia de generar espacios pedagógicos que propicien el desarrollo de la reflexividad como competencia central de la formación de un músico; la actitud reflexiva frente a las situaciones problemáticas de las prácticas, es la que asegura la capacidad para seguir creciendo musical y técnicamente de una manera autónoma.

En línea con este pensamiento, Pablo Vain (2003) discute la importancia de encontrar un balance entre el desarrollo de una racionalidad técnica que resuelve los problemas a partir 
de soluciones generales, comunes y "preconcebidas" y la competencia según la cual el profesional puede discernir al interior de lo que se define como "zonas indeterminadas". Estas situaciones son de un orden complejo y no son "abordables" desde una postura reduccionista e inmediata, lo que hace indispensable desarrollar en los estudiantes una visión reflexiva y crítica que les permita abordar las situaciones inciertas que emergen en su ejercicio como instrumentistas y músicos de manera creativa y personal. Se espera en este sentido, que la diversidad de opciones musicales propuestas por este estudio para trabajar los diversos problemas detectados represente un material adecuado para acompañar y potenciar el ejercicio de reflexión por parte de estudiantes y profesores. Reflexividad que se expresa en la capacidad para detectar los elementos claves de las zonas problemáticas de la práctica así como los múltiples caminos para resolverlas.

\section{PROBLEMA ABORDADO Y OBJETIVOS DEL ESTUDIO}

La cátedra de guitarra clásica del Departamento de Música de la PUJ se inscribe dentro de un sistema de formación musical que articula los niveles infantiles y juveniles (Programa Infantil y Juvenil de Música) con un nivel de formación profesional a nivel de pregrado y posgrado (Carrera de Estudios Musicales y Maestría en Música, respectivamente). Dentro del desarrollo de la cátedra de guitarra al interior de este sistema, así como del contexto pedagógico y musical en el que acontece, se evidencian algunas debilidades que fueron objeto de estudio del presente proyecto, con miras a una propuesta metodológica y didáctica que ofreciera espacios pedagógicos alternativos de crecimiento y articulación entre los diversos niveles del sistema de formación (infantil-juvenil-adulto/profesional) en los planos técnico y musical.

De otra parte, se observó dentro del estado del arte de métodos y textos relacionados con el aprendizaje de la guitarra, una tendencia a dividir el desarrollo en etapas lineales, cuando lo que se observa en la práctica es una pluralidad de procesos individuales y complejos de formación que parecen funcionar más bien a manera de espiral (de lo sencillo a lo complejo y de regreso a lo sencillo).

En este marco de ideas, se detectaron los siguientes aspectos problemáticos:

- La ausencia de una sistematización explícita de las dificultades instrumentales que se presentan en el énfasis de guitarra de la PUJ.

- La falta de una recolección sistematizada, accesible y pertinente de material didáctico y musical que aporte a la resolución de dificultades instrumentales diversas.

- La poca inclusión de música tradicional latinoamericana y de música con lenguajes y grafías no convencionales para distintos niveles en los métodos de guitarra clásica desarrollados en Latinoamérica en los siglos XX y XXI.

- $\quad$ Ausencia de material pedagógico que ofrezca ejercicios técnicos específicos conectados de manera explícita con el repertorio musical y que se pueda abordar desde cualquier nivel de formación.

En línea con estas problemáticas, el estudio tuvo como base el siguiente objetivo general: Desarrollar un material pedagógico de carácter reflexivo, orientado al aprendizaje del guitarrista clásico, que incluya referencias de la tradición folclórica latinoamericana, los lenguajes musicales de vanguardia y el repertorio clásico. 
Los objetivos operativos o específicos que hicieron posible el desarrollo de este material fueron los siguientes:

- Identificar las principales dificultades técnicas y musicales que aparecen a lo largo del proceso de desarrollo instrumental.

- Recopilar y componer ejercicios y repertorio para trabajar las dificultades específicas.

- Organizar el material recopilado y compuesto en un sitio virtual a partir de las categorías encontradas.

\section{METODOLOGÍA}

La metodología que se utilizó para el desarrollo del proyecto fue de carácter cualitativo y constó de las siguientes etapas: identificación de dificultades, recopilación y composición de piezas y ejercicios, sistematización y organización del material de acuerdo a categorías de dificultades y de estilos.

La detección de dificultades se desarrolló de la siguiente manera: cada uno de los tres docentes - investigadores tomó nota de las dificultades emergentes en los procesos de los estudiantes desde la experiencia pedagógica propia. Estas dificultades se organizaron en cuatro grandes categorías: mano derecha, mano izquierda, técnicas extendidas y "otras dificultades". A partir de estos hallazgos se elaboró una tabla que organiza en una secuencia numérica las dificultades por categoría general. Esta tabla fue la guía para la codificación de las piezas que se presenta más adelante, en la sección de análisis y resultados. Las piezas seleccionadas son fruto de una indagación dentro del repertorio para guitarra, dando especial énfasis a la música tradicional latinoamericana y a la música escrita para guitarra por compositores y guitarristas del Departamento de Música de la PUJ. Esta indagación tenía como finalidad encontrar pasajes específicos del repertorio en los que aparecen las dificultades detectadas durante la primera etapa del estudio.

Es importante aclarar en este punto que cuando se aborda una categoría o dificultad específica, de inmediato surge la posibilidad de mencionar una gran cantidad de literatura existente, citar pasajes de obras determinadas como contextos para trabajar las dificultades, y de indagar sobre explicaciones ofrecidas por parte de los compositores e intérpretes consultados. Esta situación implicó tener un sentido crítico que permitiera a los investigadores elegir el material que realmente es pertinente dentro de la gran diversidad de opciones disponibles. Es por eso que la propuesta incluye sugerencias de links o bibliografía para consulta complementaria y/o profundización, con el fin de que el usuario del material pueda acceder al inmenso mundo de información guitarrística existente a través de la historia y que se encuentra disponible en libros, audios o videos.

De otra parte, se compusieron ejercicios y piezas que permitieran trabajar de manera explícita las dificultades planteadas por el proyecto. En resumen, se reunió material musical de dos tipos para trabajar las dificultades específicas: material ya compuesto para guitarra por otros compositores y material compuesto por los autores del presente proyecto (ejercicios y estudios).

Finalmente, se realizó una clasificación del material recopilado y compuesto de acuerdo a las categorías de dificultades definidas, así como a los formatos (música solista o de cámara) y estilos de las piezas trabajadas. El corpus musical fue organizado en un site de Google, al cual se hará alusión más adelante en este artículo. 


\section{ANÁLISIS DE LA INFORMACIÓN Y RESULTADOS DEL PROYECTO}

\section{Problemas encontrados por categoría}

Como se mencionó en la sección de metodología, los problemas o dificultades que se encontraron a lo largo del proceso de investigación, fueron organizados en cuatro grandes categorías:

- Mano derecha

- Mano izquierda

- Técnicas extendidas

- $\quad$ Otras dificultades

A continuación se presenta una tabla que ilustra de manera detallada las dificultades particulares que fueron asociadas a estas cuatro categorías generales.

Tabla 1. Relación de dificultades por categoría general

\begin{tabular}{|c|c|c|c|c|}
\hline & Mano Derecha (MD) & Mano Izquierda (MI) & Técnicas extendidas (TE) & Otras \\
\hline 1 & $\begin{array}{l}\text { Alternancia i, m¹; m, a; i, a: } \\
\text { libre y apoyado }\end{array}$ & Dedo pivote & Percusión & Primera vista \\
\hline 2 & Pulgar & $\begin{array}{l}\text { Desplazamientos trastes } \\
\text { I-III, I-V }\end{array}$ & Rasgueados/apagados & \\
\hline 3 & Canto anular & Cejilla parcial & Tambora & \\
\hline 4 & $\begin{array}{l}\text { Pulgar alterna con i, con m, } \\
\text { con a }\end{array}$ & Cejilla entera & Pizzicato & \\
\hline 5 & Pulgar alterna con im, ia, ma & Contracción corta & Pizz. Bartók & \\
\hline 6 & Pulgar alterna con ima & Distensión corta & Tap (ambas manos) & \\
\hline 7 & $\begin{array}{l}\text { Pulgar simultáneo con i, con } \\
\text { m, con a }\end{array}$ & $\begin{array}{l}\text { Ligados ascendentes con } \\
\text { cuerdas al aire }\end{array}$ & Bend & \\
\hline 8 & $\begin{array}{l}\text { Pulgar simultáneo con im, } \\
\text { ia, ma }\end{array}$ & $\begin{array}{l}\text { Ligados ascendentes sin } \\
\text { cuerdas al aire }\end{array}$ & Glissandi & \\
\hline 9 & Pulgar simultáneo con ima & $\begin{array}{l}\text { Ligados descendentes } \\
\text { con cuerdas al aire }\end{array}$ & Armónicos naturales & \\
\hline 10 & $\begin{array}{l}\text { Destacar distintas voces } \\
\text { al momento de tocar con } \\
\text { dedos simultáneos }\end{array}$ & $\begin{array}{l}\text { Ligados ascendentes con } \\
\text { cuerdas al aire }\end{array}$ & $\begin{array}{l}\text { Otros efectos } \\
\text { representados con grafía no } \\
\text { convencional }\end{array}$ & \\
\hline 11 & Arpegio & Escalas & & \\
\hline 12 & $\begin{array}{l}\text { Pulgar alterna con ima sobre } \\
\text { una sola cuerda }\end{array}$ & Posiciones fijas & & \\
\hline 13 & & Traslados verticales & & Improvisación \\
\hline $1 \mathrm{~A}$ & im, ma, ia simultáneos & Vibrato & & \\
\hline 1B & ima simultáneos & Preparación & & \\
\hline 14 & Contrastes tímbricos & & & \\
\hline
\end{tabular}


Posteriormente, cada uno de los investigadores utilizó algún tipo de procedimiento para clasificar las piezas y los fragmentos de acuerdo al tipo de dificultad. El proceso de búsqueda de material musical para trabajar las dificultades o problemas específicos se reflejó en una tabla (Ver Tabla 2) que incluye cuatro columnas: Nombre de la pieza, autor, número(s) de compás(es) (en donde aparece la dificultad) y la dificultad específica que aparece en el pasaje elegido. La dificultad específica se registraba en la tabla mediante un código que representaba de una parte la categoría general: "MD" - Mano derecha, "MI" - Mano Izquierda, "TE" - Técnicas Extendidas y "Otros". De otra parte, el código incluía un número que correspondía a la dificultad puntual de acuerdo a la sistematización de dificultades relacionada en la Tabla 1 (por ejemplo: "MD 4" corresponde a la "Dificultad número 4 de la categoría "Mano Derecha", es decir: "Pulgar alterna con i, con m, con a").

Tabla 2. Codificación de dificultades en las piezas musicales

\begin{tabular}{|c|c|c|c|c|c|c|c|}
\hline Pieza & Autor & Compases & Problema & Pieza & Autor & Compases & Problema \\
\hline Janyama & Sebastián & $79-98$ & MD 4 & Los Pollitos & César Rojas & $13-$ & TE $1,3,6,8$ \\
\hline Meavai & Villanueva & & & Decían & & & \\
\hline \multirow[t]{2}{*}{ Secta Olivo } & Eblis & 1 a 6 & MD 5 & Guitarra & Rodolfo & & \\
\hline & Álvarez & & & Naranja & Acosta & & \\
\hline Batalla & Eblis & 1 a 18 & MI 9 y 10 & Imagen & Marco & P61 C 1 & TE10 \\
\hline Cráneo & Álvarez & & & Rota & Suarez & & \\
\hline Batalla & Eblis & $19 a$ & MD 13 & Imagen & Marco & P61 S3 & TE10 \\
\hline Cráneo & Álvarez & & & Rota & Suarez & & \\
\hline \multirow[t]{2}{*}{ Estudio 1} & Andrés & 1 a 18 & MD 1 & Imagen & Marco & P64 S2-3 & TE7 \\
\hline & Samper & & & Rota & Suarez & & \\
\hline \multirow[t]{2}{*}{ Estudio 1} & Andrés & 1 a 18 & MD 8 & Imagen & Marco & P63S1 & TE10 \\
\hline & Samper & & & Rota & Suarez & & \\
\hline \multirow[t]{2}{*}{ Danza } & Andrés & 15 a 32 & TE 6 & Imagen & Marco & P63 S3 & TE10 \\
\hline & Samper & & & Rota & Suarez & & \\
\hline \multirow[t]{2}{*}{ Danza } & Andrés & 33 a 51 & MD 5 & Imagen & Marco & P68 S2 & TE 10 \\
\hline & Samper & & & Rota & Suarez & & \\
\hline \multirow[t]{2}{*}{ Danza } & Andrés & 77 а 91 & MD 11 & Imagen & Marco & P64 S1 & TE 10 \\
\hline & Samper & & & Rota & Suarez & & \\
\hline Calma y & $\mathrm{JC}$ & 2 a 29 & MD 11 & Imagen & Marco & P67 S2 & TE 10 \\
\hline \multirow[t]{3}{*}{ Turbulencia } & Marulanda & & & Rota & Suarez & & \\
\hline & & & & Imagen & Marco & P68 S3 & TE 10 \\
\hline & & & & Rota & Suarez & & \\
\hline
\end{tabular}

\section{MATERIAL RECOPILADO Y ESCRITO PARA EL PROYECTO}

Los problemas técnicos detectados durante la recopilación de información sirven como eje central para la propuesta final que combina compilaciones de repertorio conocido y material inédito. Dentro de esta recopilación de repertorio vale la pena resaltar los materiales inéditos: de una parte obras escritas por compositores y guitarristas del programa de música de la PUJ, y de otra, ejercicios y estudios escritos por los tres investigadores. Estas obras pasan a 
enriquecer el material de consulta disponible para los estudiantes de guitarra de los programas de la Facultad de Artes de la PUJ.

A la vez, se destaca la propuesta de improvisación incluida en algunas piezas que busca generar inquietud en los estudiantes en cuanto al ejercicio creativo en tiempo real. No solo como una herramienta para ayudar al proceso de aprendizaje instrumental, sino también como una rutina que puede ser incorporada en la práctica de la guitarra, y que permite entender el quehacer musical como un proceso orgánico y circular, en el que la interpretación y la creatividad se nutren entre sí enriqueciendo la expresividad y la relación misma del intérprete con el sonido.

Se destaca también la inclusión de un estudio original y varias piezas que utilizan grafías no convencionales, así como la inclusión de ejercicios y un estudio original para el desarrollo de la capacidad de lectura a primera vista en el instrumento.

El repertorio, constituido por ejercicios, estudios y piezas, fue almacenado y organizado de manera sistemática en dos sites de Google que permiten hacer búsquedas a través de los menús y sub menús del site o mediante el motor de búsqueda de la plataforma. De esta manera, si al usuario le interesa por ejemplo, encontrar ejercicios y/o repertorio relativos a la dificultad "ligados ascendentes" podrá buscar directamente la categoría en el menú correspondiente a "Mano izquierda" o escribir las dos palabras ("ligados ascendentes") en el motor de búsqueda de la plataforma. En este segundo caso, la plataforma desplegará todas las subpáginas que contienen información relativa a la dificultad consultada.

Cada página contiene una serie de piezas o ejercicios para los cuales hay indicaciones o recomendaciones sobre cómo abordar el material. En algunos casos, se incluye alguna lectura relacionada con el material musical (como en el caso de la sección de improvisación). Los sites en los que se recoge el material son los siguientes:

Site de dificultades o problemas

\section{https://sites.google.com/site/proyectoguitarrapuj/}

La página de inicio de este site contiene los siguientes títulos:

- Recomendaciones generales

- Mano derecha

- Mano izquierda

- Efectos en la guitarra

- Improvisación

- Lectura a primera vista

- $\quad$ Repertorio - Conexión con el site de Repertorio (Ver el apartado siguiente)

- Vínculos de interés - Conexión con otros sitios de partituras para guitarra y con plataformas musicales (e.g. programas de radio relacionados con la guitarra). 


\section{https://sites.google.com/site/repertproyectoguitarrafinal/}

Este site recoge todo el material de piezas y estudios que fue recopilado para el proyecto. Se organiza de la siguiente manera:

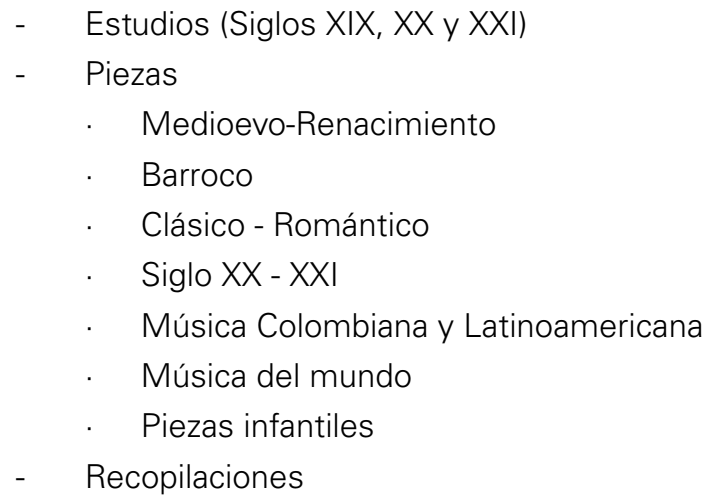

\section{CONCLUSIONES}

A manera de cierre podemos señalar dos campos en los que se agrupan los aportes más importantes del proyecto. En primer lugar, contribuyó a nuestro propio proceso como pedagogos, pues nos permitió desplegar un ejercicio reflexivo valioso con relación a nuestras propias prácticas docentes y a las principales dificultades de orden instrumental y musical que allí emergen. La posibilidad, de otra parte, de generar esta reflexión como equipo nos permitió intercambiar ideas, metodologías, estrategias, material y posturas frente al proceso general de enseñanza de la guitarra.

En segundo lugar, el producto final cumple con las expectativas que se tenían en un principio de proponer un corpus de material que tuviera las siguientes características: que integrara la expresión musical con el desarrollo técnico, que fuera susceptible de ser abordado de manera "holística" —no lineal— según las necesidades de quien lo utilice y sin importar su nivel de formación, y que incorporara material latinoamericano con un énfasis particular en la producción del Departamento de Música de la PUJ. Finalmente, que ofreciera una opción de espacios para el desarrollo de habilidades y saberes en áreas poco exploradas por los métodos tradicionales de instrumento como la lectura a primera vista, la improvisación y el uso de grafías no convencionales. En este sentido, puede ser un aporte valioso para el medio de la guitarra por la originalidad de su estructura y de su contenido.

Es importante, sin embargo, hacer claridad sobre un asunto: Teniendo en cuenta que el desarrollo instrumental de cada músico va íntimamente ligado y depende estrechamente de su desarrollo personal y de su ámbito cultural, este material de apoyo al aprendizaje no podrá remplazar jamás el contacto presencial entre el profesor y el alumno. La relación interpersonal permanecerá siempre como un espacio único en el que las circunstancias del momento tienen un papel importante en el desarrollo de la clase y de la manera de sugerir y guiar que puede asumir el maestro con respecto al estudiante. Las circunstancias individuales de formación intelectual, entorno sociocultural y conformación física del guitarrista, hacen de cada individuo un universo único e irrepetible que solamente con la clase presencial puede ser apreciado en 
su totalidad. Por eso, una de las dificultades inherentes al formato virtual es no poder asistir personalmente a la formación del guitarrista. Se entiende entonces este producto más como un apoyo para los procesos pedagógicos que como un remplazo de la clase de instrumento. Cuando no existe la mediación explícita de un docente se debe suponer que el interesado tiene la claridad y conciencia suficientes para afrontar por sí mismo y de manera autocrítica cualquier problema que se presente en el camino, haciendo uso de las "Herramientas Didácticas para Guitarristas".

Para finalizar, vale la pena mencionar que el material presenta un potencial interesante para seguir desplegándose, nutrido por los aportes que irán haciendo a través del tiempo guitarristas y maestros del instrumento de otras latitudes y culturas; así como por los mismos autores del proyecto. Esta ampliación del corpus del proyecto no solamente tiene que ver con aumentar la cantidad de material y diversificar el repertorio recopilado sino también con la posibilidad de incorporar soportes alternativos para el registro musical, diferentes a la partitura, como es el caso de archivos de audio y de video.

Futuros proyectos de investigación pueden indagar sobre los siguientes temas: ampliación del corpus pedagógico recopilado, inclusión de piezas compuestas por los estudiantes en el repertorio recopilado, seguimiento al impacto pedagógico del uso de esta herramienta en ámbitos formales y no formales de enseñanza de la guitarra, experiencias con recopilaciones similares en otras áreas instrumentales, entre otros.

\section{NOTAS}

1 "i" corresponde a dedo índice; "m" a dedo medio o mayor; "a" a dedo anular.

\section{REFERENCIAS}

Aguado, Dionisio. "Nuevo método de guitarra Op 6". Documento de dominio público disponible en Internet. 1843.

Camilloni, Alicia R.W. de. La evaluación de los aprendizajes en el debate didáctico contemporáneo. Buenos Aires: Paidós, 1998.

Carlevaro, Abel. Serie didáctica para guitarra. Última edición. Volumen 1 a 4. Buenos Aires: Barry, 2006.

Carulli, Ferdinando. Método completo para guitarra. Paris: Henry Lemoine, 1955.

Fernández, Eduardo. Técnica, mecanismo, aprendizaje. Una investigación sobre cómo llegar a ser guitarrista. 1 ra edición. Montevideo: Ediciones ART, 2000.

Gangi, Mario. Metodo per chitarra. Volumen 1 a 3. 1 ra edición Buenos Aires: Ricordi, 1971.

Giuliani, Mauro. “Estudios para guitarra". Op 1, Op 48, Op 98. [En línea] http://maurogiuliani.free.fr/ en/ (Acceso: mayo de 2015).

Pujol, Emilio. Escuela razonada de la guitarra, basada en los principios de la técnica de Tárrega. Tres volúmenes. Buenos Aires: Ricordi Americana, 1954. 
Roch, Pascual. Método moderno para la guitarra. 1ra edición. Volumen 1 a 3. Nueva York: G. Schirmer, 1921.

Shearer, Aaron. Learning the classical guitar. Parte 1 a 3. Mel Bay Publications, Inc. Pacific, 1990.

Sor Fernando. Complete works for guitar. 3 vols. Edited by Brian Jeffery. Second edition, London: Tecla, 1994.

Sor, Fernando. Método para la guitarra. Paris, Publicado por A. Merrick. Documento de dominio público disponible en Internet. 1832.

Vain, Pablo "El Diario Académico: una estrategia para la formación de docentes reflexivos". Perfiles Educativos; Universidad Nacional Autónoma de México. Vol. XXV, núm. 10 (2013): 56-68.

\section{Cómo citar este artículo:}

Posada Ramos, Carlos; Quevedo Barrero, César y Samper

Arbeláez, Andrés. “Herramientas didácticas para el desarrollo técnico musical del guitarrista clásico a través de ejes problemas". Cuadernos de Música, Artes Visuales y Artes Escénicas, 11(1), 39-53, 2016. http://dx.doi.org/10.11144/ Javeriana.mavae11-1.hddt 
